\section{Déficit en alphat-antitrypsine et cancer primitif du foie}

L'alpha ${ }_{1}$-antitrypsine, une glycoprotéine synthétisée par le foie, est la principale anti-protéase du sérum humain. Sa propriété biologique principale paraît être de lier et d'inactiver l'élastase leucocytaire. Son intérêt, pour les médecins, est venu de la mise en évidence, en 1965 , d'une association entre le déficit héréditaire en alpha ${ }_{1}$-antitrypsine et l'emphysème pulmonaire d'une part, la cirrhose d'autre part. La synthèse hépatique de l'alpha ${ }_{1}$ antitrypsine est en effet contrôlée par une paire de gènes situés sur le locus Pi. Plus de 25 allèles de ces gènes ont été identifiés. Certains, notamment ceux dénommés $S$ et $Z$, sont responsables d'une synthèse et d'une sécrétion anormales de la protéine, avec pour résultat une concentration plasmatique abaissée. Ainsi, les sujets homozygotes (phénotypes PiSS ou PiZZ) ont une concentration plasmatique à $10-15 \%$ de la normale, et les hétérozygotes (phénotypes PiMS ou PiMZ par exemple, $M$ étant le gène normal habituel) une concentration à 50-60\% de la normale. Bien que l'association du déficit héréditaire avec une cirrhose et un cancer primitif du foie ait été bien documentée dans le passé, le risque, par rapport à une population normale, n'est pas connu.

Eriksson et coll. (qui avaient découvert l'association avec l'emphysème et la cirrhose) ont tiré parti des facilités offertes par la ville de Malmö, en Suède, pour quantifier ce risque [I]. Malmö est une ville d'environ 240000 habitants qui comporte un hôpital général avec un service central d'anatomie pathologique. Une méthode standardisée d'autopsie a été introduite en 1958 afin de faciliter les études épidémiologiques. Entre 1963 et 1982 , période choisie pour l'étude, 35280 autopsies ont été effectuées, pour 5I 730 décès, soit $68,2 \%$ de l'ensemble des décès, et plus de $85 \%$ des décès à l'hôpital. Pendant cette période, grâce aux dossiers médicaux des services hospitaliers concernés, tous les habitants de Malmö ayant un déficit homozygote PiZZ ont été identifiés : 20 de ces habitants sont morts pendant la période d'étude et $\mathbf{I} 7$ ont été autopsiés. Pour chaque homozygote, quatre sujets témoins (également autopsiés), du même sexe et d'âge le plus proche possible, ont été sélectionnés. Connaissant leur fréquence dans la population (I/666), 2 I homozygotes étaient attendus : fait remarquable pour une étude de ce type, 20 ont été trouvés. Les constatations principales faites dans l'étude sont : (a) une très forte relation entre déficit homozygote et cirrhose, avec un risque relatif multiplié par 7,8 en moyenne chez les homozygotes (intervalle de confiance : 2,4 à 24,7 ); (b) une très forte relation entre déficit homozygote et carcinome hépatocellulaire, avec un risque relatif multiplié par 20 en moyenne chez les homozygotes (intervalle de confiance 3,5 à I I 4,3). Quand les résultats ont été "stratifiés " en fonction du sexe, le risque n'était augmenté de façon significative que chez les hommes. La raison de cette différence n'est pas claire.

Cette étude démontre de façon non ambiguë un risque très élevé de cirrhose et de carcinome hépatocellulaire chez les sujets ayant un déficit homozygote. Jusqu'à présent, parmi les maladies métaboliques héréditaires, seules l'hémochromatose et la tyrosinémie étaient connues pour avoir un risque aussi élevé. Ces constatations posent évidemment le problème du mécanisme de la cancérisation. On sait que, dans la majorité des cas, le carcinome hépatocellulaire est associé à une infection par le virus de l'hépatite B. Apparemment, chez cinq des huit malades ayant une cirrhose, l'antigène HBs était absent du sérum; chez trois de ces cinq malades, l'ADN du virus $B$ a pu être cherché dans le foie par hybridation moléculaire : il était absent. D'autres facteurs que le virus B pourraient donc être en cause. S. E.

1. Eriksson S, Carlson J, Velez R. Risk of cirrhosis and primary liver cancer in alpha ${ }_{1}$-antitrypsin

\section{Antigène $H-Y$ et spermatogenèse}

La masculinité chez les mammifêres est liée à la présence d'un chromosome Y. On ignore par contre quel est l'élément, porté par l'Y, qui joue un rôle décisif dans la différenciation testiculaire. On connaît depuis longtemps un antigène de surface spécifique du mâle, l'antigène $\mathrm{H}-\mathrm{Y}$ (Histocompatibilité-Y), qu'on a considéré comme étant l'inducteur de la différenciation sexuelle. Or, des expériences datant de 1984 semblent éliminer cette hypothèse.

McLaren et coll. à Londres [I] ont utilisé des soutis Sxr. Comme médecine/sciences l'a déjà mentionné ( $n^{\circ} 3$, vol. 2, p. 157), il s'agit de souris dont le chromosome $Y$ porte un élément sur son bras court qui peut se transloquer sur le bras court du chromosome $\mathrm{X}$ au cours de la méiose et qui impose le caractère mâle aux animaux qui en sont porteurs. On obtient ainsi des souris XX Sxr, qui sont des mâles stériles (Sxr : réversion du sexe). Ces souris portent l'antigène $\mathrm{H}-\mathrm{Y}$. Or, les auteurs ont trouvé une souris mutante qu'ils ont appelée $S_{x r}$, qui possédait le caractère mâle, mais était dépourvue d'antigène $\mathrm{H}-\mathrm{Y}$, lequel n'est donc probablement pas le déterminant primaire du sexe. Quel pourrait donc être le rôle de cet antigène, dont la présence dans toutes les cellules mâles rend improbable le fait qu'il puisse n'en jouer aucun? L'hypothèse la plus vraisemblable est qu'il intervienne dans la spermatogenèse, mais sa démonstration se heurte à de grandes difficultés. L'utilisation des Sxr habituels est impossible, car la présence de deux chromosomes X semble incompatible avec le maintien de cellules germinales au-delà de la période périnatale.

Burgoyne et coll. [2] ont eu l'idée d'avoir recours à des souris XO Sxr, qui certes sont stériles, mais qui présentent presque tous les stades de la spermatogenèse, à l'exception des tout derniers. Elles possèdent aussi l'antigène $\mathrm{H}-\mathrm{Y}$. Ils ont pu 
produire ensuite des souris $\mathrm{XO}$ Sxr', porteuses de la mutation qui fait disparaître H-Y. Leur spermatogenèse est beaucoup plus sévèrement atteinte que celle des XO Sxr, et ne dépasse pas le stade de la spermatogonie. Les auteurs rappellent en outre qu'une souris mâle $\mathrm{XO}$, à spermatogenèse déficiente et dépourvue d'antigène $\mathrm{H}-\mathrm{Y}$, avait été décrite en 1977 et était restée unique.

Peut-on appliquer ces données à l'espèce humaine? Les hommes XX (voir médecine/sciences $n^{\circ} 6$, vol. I, p. 33I) ont, dans la plupart des cas, acquis le facteur masculinisant par transfert de séquences provenant du bras court du chromosome Y. On a décrit un gène de spermatogenèse chez l'homme, probablement homologue de celui de la souris; il est porté par le bras long de $\mathrm{Y}$ et ne devrait donc pas être transféré chez les hommes XX. Or, la recherche de l'antigène $\mathrm{H}-\mathrm{Y}$ s'est montrée négative chez les cing sujets mâles XX qui ont été testés jusqu'à présent. Ces résultats confirment l'hypothèse que, si l'antigène $\mathrm{H}-\mathrm{Y}$ n'est pas le déterminant primaire du sexe, il est un agent indispensable à la spermatogenèse.

J.-C. D.

I. McLaren A, Simpson E, Tomonari $K$, Chandler $P$, Hogg $H$. Male sexual differentiation in mice lacking $\mathrm{H}-\mathrm{Y}$ antigen. Nature $1984 ; 312$ : 552-5.

2. Burgoyne PS, Levy ER, McLaren A. Spermatogenic failure in male mice lacking $\mathrm{H}-\mathrm{Y}$ antigen. Nature 1986; 320: 170-2.

I. Ruoslahti E, Pierschbacher MD. Arg-Gly-Asp: a versatile cell recognition signal. Cell 1986; 44: 517-8.

2. Jenne D, Stanley K. Molecular cloning of S-protein, a link between complement, coagulation and cell-substrate adhesion. EMBO 7 1985; 4: 3153-7.

3. Auffrey C. Un modèle moléculaire de l'interaction entre l'antigène $T_{4}$ et les antigènes HLA de classe II ou le virus LAV. $C R$ Acad Sci Paris 1986; 302: 287-92.

4. Shirakami A, Shigekiyo T, Hirai Y, et al. Plasma fibronectin deficiency in eight members of one family. Lancet 1986; i: 473-4.

\section{Un signal polyvalent de reconnaissance cellulaire}

Un article de Ruoslahti et Pierschbacher (La Jolla, Californie) paru dans Cell [I] passe en revue les propriétés surprenantes d'une séquence de trois acides aminés dans les réactions de reconnaissance entre des protéines qui la contiennent et la surface des cellules. Le modèle de ces protéines est la fibronectine*, glycoprotéine de grande taille, dont le site d'attachement a été isolé, séquencé, et reproduit par synthèse chimique. En raccourcissant progressivement ces peptides de synthèse, on voit qu'au sein de cette protéine de 2500 acides aminés une séquence de trois acides aminés suffit comme signal de reconnaissance. Ce tripeptide, arginine-glycocolle-acide aspartique ou $\mathrm{RGD}^{* *}$ ne peut être modifié sans perdre ses fonctions. Les peptides de synthèse contenant RGD se fixent directement aux cellules lorsqu'ils sont insolubles. S'ils sont solubles, ils inhibent la liaison de la fibronectine aux cellules.

La fibronectine n'est pas restée seule à posséder de telles propriétés. Le fibrinogène contient deux séquences RGD et le facteur Willebrand une, et leur fixation aux plaquettes est empêchée par les peptides de synthèse à RGD. Une autre protéine munie de cette séquence est la vitronectine, le composant actif du facteur d'étalement du sérum. La vitronectine est identique à la protéine $S$, qui forme des complexes avec certains composants du complément et semble également un précurseur de la somatomédine B [2]. Les séquences RGD présentes dans les protéines adhésives sont reconnues par des récepteurs à la surface des cellules. Trois types en ont été identifiés jusqu'à présent. Les deux premiers ont été isolés de cellules d'ostéosarcome humain et de fibroblastes de rat. Ils sont formés chacun de deux chaînes, de taille supérieure à 100000 daltons. L'un est spécifique de la fibronectine, l'autre de la vitronectine. Le troisième, extrait des plaquettes sanguines, présente une spécificité moins étroite, puisqu'il peut se lier à fibronectine, vitronectine, fibrinogène et facteur Willebrand. D'autres récepteurs sont en cours d'isolement, capables notamment de lier le collagène et certains facteurs de croissance.

L'existence de récepteurs différents montre que, si la séquence RGD est nécessaire, elle ne suffit pas à la fixation de toute molécule qui la contient. Deux explications sont proposées. Le tripeptide pourrait être nécessaire comme point d'ancrage, mais la fixation réclamerait un autre site de liaison, spécifique de chaque protéine. Dans l'autre hypothèse, l'information contenue dans RGD serait suffisante, mais les séquences qui l'entourent lui imprimeraient une conformation qui ne s'adapte qu'à certains récepteurs. Les conséquences de cette découverte sont considérables. Dans leur généralité d'abord, car on a trouvé le tripeptide dans des protéines du colibacille et d'une amibe, Dict yostelium discoideum. Pour la différenciation ensuite, au cours de laquelle la capacité de nombreuses cellules à se lier avec des molécules de la matrice varie. Le système de reconnaissance RGD pourrait donc servir de signal de reconnaissance à usages multiples.

Ajoutons enfin qu'en France Charles Auffrey [3] vient de montrer l'importance d'un analogue du RGD où le glycocolle est remplacé par une phénylalanine. Il est présent notamment dans les chaînes $\beta$ des antigènes HLA de classe II, ainsi que dans la protéine $F$ du virus du SIDA, et pourrait jouer un rôle dans l'interaction du virus $\mathrm{LAV}$, comme des antigènes HLA, avec l'antigène $\mathrm{T}_{4}$.

J.-C.D.

* La fibronectine est présente dans le plasma à un taux de 300 à $400 \mathrm{mg}$ par litre. Signalons à cette occasion qu'un déficit familial en fibronectine vient d'être décrit pour la première fois (au fapon) [4]

** Il existe deux nomenclatures pour désigner les acides aminés, une usuelle à trois lettres, une courte à une seule lettre : $A r g=R ; G l y=G ; A s p=D$.
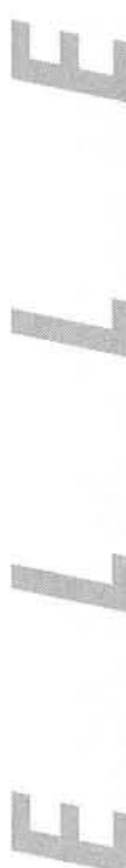